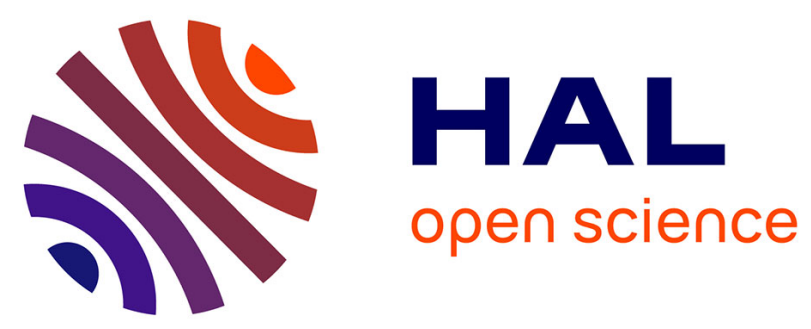

\title{
Shock-induced cavitation as a way of accelerating phenol oxidation in aqueous media
}

Hugo Dutilleul, Anthony Partaloglu, Patrick da Costa, Maria Elena Gálvez

\section{To cite this version:}

Hugo Dutilleul, Anthony Partaloglu, Patrick da Costa, Maria Elena Gálvez. Shock-induced cavitation as a way of accelerating phenol oxidation in aqueous media. Chemical Engineering and Processing:

Process Intensification, 2017, 112, pp.47-55. 10.1016/j.cep.2017.01.003 . hal-01447908

\section{HAL Id: hal-01447908 \\ https://hal.sorbonne-universite.fr/hal-01447908}

Submitted on 27 Jan 2017

HAL is a multi-disciplinary open access archive for the deposit and dissemination of scientific research documents, whether they are published or not. The documents may come from teaching and research institutions in France or abroad, or from public or private research centers.
L'archive ouverte pluridisciplinaire HAL, est destinée au dépôt et à la diffusion de documents scientifiques de niveau recherche, publiés ou non, émanant des établissements d'enseignement et de recherche français ou étrangers, des laboratoires publics ou privés. 


\section{Shock-induced cavitation as a way of accelerating phenol oxidation in aqueous media}

Hugo Dutilleul, Anthony Partaloglu, Patrick Da Costa, Maria Elena Galvez*

Sorbonne Universités, UPMC, Univ. Paris 6, CNRS, UMR 7190, Institut Jean Le Rond d'Alembert, 2 place de la gare de ceinture, 78210 Saint-Cyr-L'Ecole, France

*Corresponding author: elena.galvez_parruca@upmc.fr, tel.: +33 (0) 130854877

\section{Graphical Abstract}

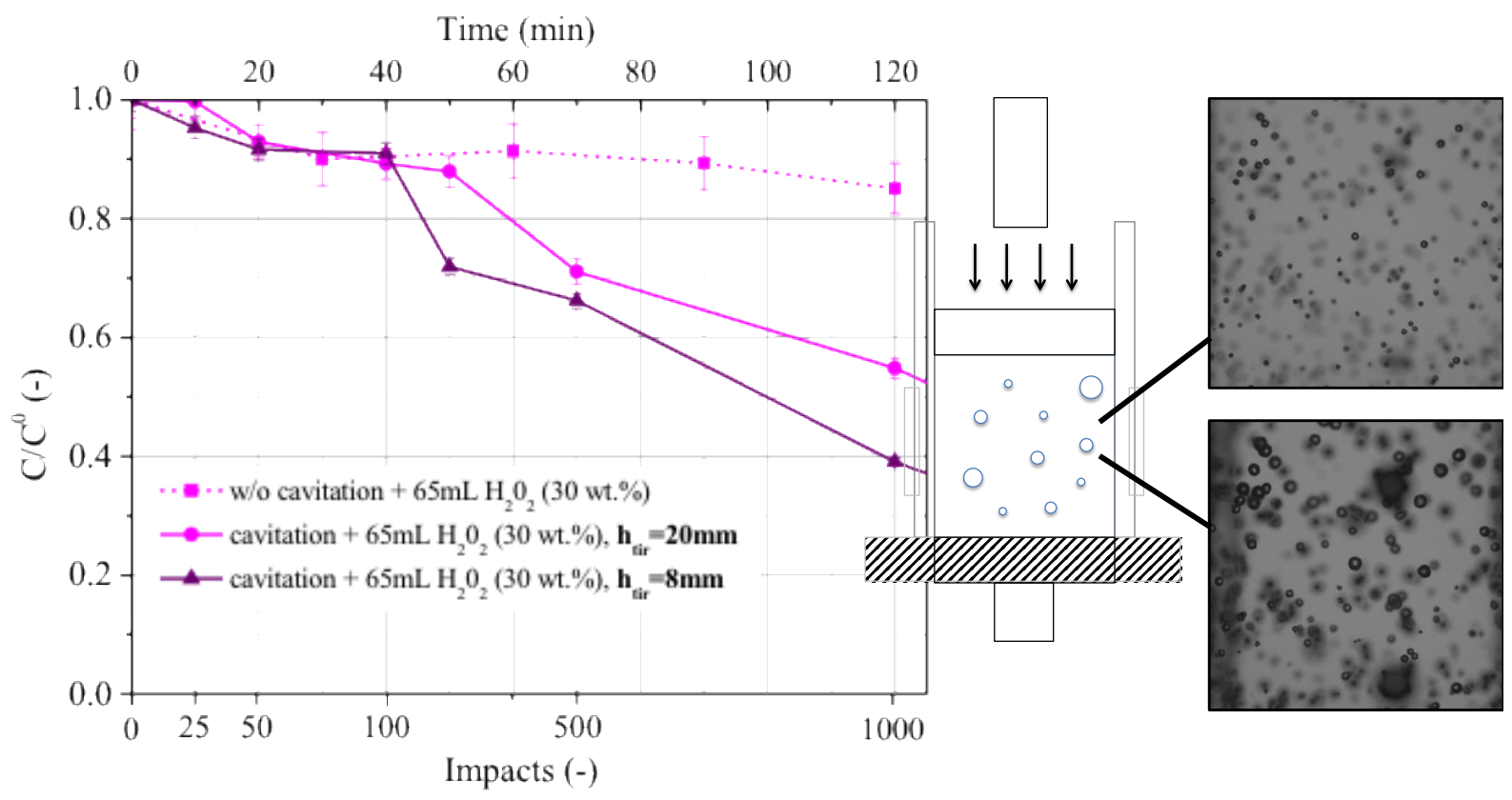




\title{
Highlights
}

- Cavitation induced by the impact of a solid piston on liquid surface

- Shock-waves of several bars amplitude exciting a wide frequency range

- Phenol degradation initiated at ambient temperature and absence of oxidants

- High phenol mineralization extents measured upon $\mathrm{H}_{2} \mathrm{O}_{2}$ addition

- Accelerated active radical formation in the presence of shock-induced cavitation

\begin{abstract}
Shock-induced cavitation phenomena, resulting from the propagation of the trail of shockwaves generated upon the impact of a solid piston on a liquid surface, was used as an innovative way of intensifying the oxidation of phenol in aqueous media. The amount of energy communicated by the impact was found to be proportional to the impact height, and was directly related to the maximal pressure attained, i.e. in the order of several tens of bars. This peak was followed by a rarefaction period that results in the origination of several cavitation phenomena, which continued upon piston rebound and further shock-wave generation and propagation. The multi-frequency nature of shock-induced cavitation, capable of exciting a wide range of frequencies between $1 \mathrm{kHz}$ and $100 \mathrm{kHz}$, was confirmed by means of wavelet analysis. Under shock-induced cavitation conditions, phenol degradation was found to be possible even in the absence of any oxidizing agent. High extents of mineralization, i.e. 45.3 and $56.2 \%$ TOC elimination, $50 \mathrm{mg} / \mathrm{L}$ solution, neutral $\mathrm{pH}$, were obtained in the presence of $\mathrm{H}_{2} \mathrm{O}_{2}$ (aprox. $10 \%$ vol.), pointing to an acceleration of the oxidation reaction based in a faster and more efficient creation of radical species.
\end{abstract}

Keywords: Sonochemistry; cavitation; phenol oxidation, AOPs; water treatment. 


\section{Introduction}

Water pollution, as a consequence of our industrial/agricultural development, jeopardizes one of our most precious and scarce resources. Worldwide actions promote a rational use of water and introduce new regulations that force the scientific and technological communities to seek for each time more efficient technologies for water treatment. Such water treatment technologies, i.e. the oxidation of recalcitrant organic pollutants in wastewaters, are a clear example of highly-energy demanding processes, due to their inherent problems of mass transfer and their sluggish reaction kinetics.

The technologies known as Advanced Oxidation Processes (AOPs) consider different approaches for the intensification of the oxidation of organic pollutants [1-2]. They are based on the intensive generation of reactive radicals, such as hydroxyls, able to oxidize the organic pollutants contained in wastewaters. Ultrasounds are frequently used in the intensification of chemical processes, among them the oxidation of organic pollutants in wastewaters in which ultrasonic sonochemistry plays an important role for several decades [3-10]. Typically induced by piezoelectric transducers [11], ultrasonic sonochemistry is based in the phenomena of cavitation, i.e. the formation of vapor nuclei when pressure falls below its vapor pressure at a constant temperature [12]. In fact, the physical and chemical effects of sonochemistry occur upon the cavitation of the bubbles generated during the rarefaction negative pressure - period of the ultrasound wave. During rarefaction the liquid continuity is broken at nucleation sites, microbubbles are formed and grow through a mechanism of rectified diffusion (unequal mass transfer of gas and vapors across the liquid-vapor interface), until they collapse upon pressure recuperation through both stable (non-inertial cavitation, cavity oscillation for several acoustic cycles) and transient (inertial, violent implosion) cavitation phenomena [13-15]. 
In the presence of a reactive media, such cavitation events contribute to the formation of radical species (active in free radical reaction mechanism, e.g. oxidation of organic pollutants in aqueous media), to the generation of local hot spots of extremely high temperatures (enhancing the pyrolytic or temperature-activated route of chemical reactions) and to accelerate mass transfer phenomena (i.e. by means of turbulence).

In an ultrasound type of excitation both the original amplitude and frequency of the ultrasound wave are unique. Frequency determines the scale (size and volume) of the system that will be excited, i.e. affected, by cavitation. Fewer but larger bubbles are preferably formed at low frequencies whereas more dense bubble clouds of smaller size are generated when working at higher frequencies. In consequence, the influence of the ultrasound frequency on the cavitation phenomena is clearly evidenced in the existing literature, though there is not a general agreement on the frequency ranges and their particular effects (enhancement of cavitation, mass transfer, sonication, formation of radical species, etc.) [5, 16-19]. The intensity of ultrasonic cavitation is moreover a function of the acoustic pressure in the liquid and of the attenuation of the longitudinal wave within it, as it propagates far away from the excitation source (transducer). Attenuation strongly depends on the frequency of the ultrasound wave, i.e. higher frequencies are attenuated more rapidly than low frequencies, or, in other words, the higher the frequency of the ultrasound, the greater must be the initial intensity - amplitude - to provide an equivalent intensity at an equivalent depth [20].

The difficulty of tailoring both frequency and amplitude at a time, together with the directional sensitive effect of ultrasound and its non-uniform volumetric energy density, are the main drawbacks of ultrasound cavitation that may complicate its scale-up [21-24]. A highintensity, bulk and multi-frequency type of excitation would be the ideal acoustic wave. The violent rupture of liquid continuity, i.e. caused through cavitation phenomena, can be as well 
induced by shock-waves, as seen for example in under water explosions [25]. Moreover, Randolph and co-workers [26] have recently reported that the mechanical shock in vials, i.e. caused by their accidental drop, induced several cavitation phenomena that promoted protein aggregation within the liquid media contained in such recipients. They further claimed that this was due to the formation of hydroxyl radicals formed as a consequence of cavitation.

Analogously, the strong impact of a piston on a liquid surface can induce a series of acoustic waves that propagates inside the bulk of the liquid media. The objective of the present work is to evaluate the possibility of using this particular type of excitation for the intensification of oxidation reactions occurring in a liquid media. With this aim, the oxidation of phenol in water was chosen as model reaction. The influence of the impact intensity was also considered.

\section{Experimental}

Shock waves were induced by the impact of a piston on the surface of the liquid contained in a stainless cylindrical reactor $(75 \mathrm{~mm}$ diameter, $13 \mathrm{~mm}$ thickness, $125 \mathrm{~mm}$ height). A piston of $75 \mathrm{~mm}$ diameter and $485 \mathrm{mg}$ weight is hold at a certain distance from the liquid surface with an electro magnet attached to an electric linear actuator. Once the piston is released it falls and reaches the liquid surface generating the impact. The evolution of static pressure in the liquid media upon this impact can be followed with two piezoelectric sensors (Kistler 601A, connected to a 5018A amplifier), respectively placed under the piston and at the bottom of the cylindrical reactor. The cylindrical reactor is moreover equipped with two glass windows, allowing the visualization of the cavitation phenomena. A CDD camera (Phantom V711) was used for recording videos during piston impact. The bottom of the cylinder is equipped with a mobile bottom, which can be displaced by the impact, acting as a secondary source of shock waves. Compressed air can be injected below the bottom of the 
reactor, reaching pressures between 1 and 6 bar. Before each experiment, the piston was captured by the electromagnet and lifted with the aid of the linear actuator. The air contained in the space in between the piston and the water surface was evacuated using a rotary vane pump (Alcaltel), until a vacuum pressure corresponding to the saturation pressure of the liquid contained in the reactor (water, in this case) was reached. A liquid volume of $500 \mathrm{~mL}$ was used in all the experiments. Figure 1 depicts the set-up used and the piston and moving bottom evolution upon impact.

The raw pressure signals were visualized and recorded in an oscilloscope (KeysightAgilent MSO7054A). In order to remove a great part of the acoustic noise and to get betterresolved signals a smoothing Gaussian filter was applied. The position of the piston during the shots was measured by the post-treatment of the video acquired with the Phantom camera. The image was focused on a sight placed on the upper external surface of the piston. The frames were treated using a Matlab code that, based on pixel brightness distinction, was able to determine the sight's position at each instant. The derivate of position in time finally yielded the piston's velocity and thus the energy transmitted during the impact. Experiments were performed for relative heights piston-liquid surface of $8,12,16$ and $20 \mathrm{~mm}$. The influence of using a moving bottom was also tested: the bottom was either fixed, or released, using in this last case a flow of compressed air at 2 and 4 bar, respectively.

Phenol oxidation in aqueous media was chosen as a model reaction in order to evaluate the possible chemical effects of the cavitation phenomena induced by the piston impact. Phenol solutions containing either $10 \mathrm{mg} / \mathrm{L}$ or $50 \mathrm{mg} / \mathrm{L}$ were prepared. $\mathrm{H}_{2} \mathrm{O}_{2}(30 \%$ VWR) was used in some experiments as oxidizing agent. Phenol concentration as a function of the number of impacts (reaction time) was followed with the aid of a gas chromatograph (Shimatzu GC2025, equipped with a Phenomenex Zebron ZB-WAX capillary column and a 
FID detector). The total organic carbon (TOC) content in the treated phenol solutions was as well analyzed (Multi N/C 2100S Analytic Jena).

\section{Results and discussion}

\subsection{Observation and characterization of piston impact and shock-induced cavitation}

Figure 2 shows the pressure signals recorded during a typical piston impact ( $4 \mathrm{~mm}$ height, moving bottom 4 bar, in this case). Very similar pressure signals were obtained using the two piezoelectric captors placed either under the piston (top) or at the bottom of the reactor. Both show a first rapid increase of the pressure, due to the impact and reaching several tens of bar, followed by a rarefaction negative pressure period, see points 2 and 3 in the piston velocity vs. time curve in Figure 2. The harmonics found upon this depression period are due to bubbles cavitating in the nearby of the pressure sensor. A second peak appears 4-5 ms after the impact that can be assigned to the rebound of the piston, finally impacting the liquid surface. This rebound peak reaches only few bars, and does not have the intensity of the previous main impact peak, but its consequences cannot be neglected. As stated before, the noise in the pressure signals and the presence of harmonics points to the occurrence of cavitation phenomena upon the piston rebound and impact.

Figure 2 also presents the time evolution of piston's velocity upon its release and during its impact. A first initial period of acceleration can be observed (1), right after piston's release. It is followed by a deceleration pressure (2) due to the formation of a thin layer at the interface between the piston and the liquid surface, probably containing a mixture of air and water vapor. Then, the piston finally impacts the surface, its velocity becoming almost zero, and rebounds (3) corresponding to a subsequent increase in velocity (4), until it reached for a second time the liquid's surface (5) and further continues to rebound (6), generating the trail of shock-waves that cause the second - and even third - pressure peaks observed in the 
pressure signals. The maximal impact peak pressure, the minimal pressures attained in the rarefaction period, the number of rebounds and their intensity, depend on the energy transmitted by the fall and impact of the piston. This will ultimately depend on the impact height, the mass of the piston and its surface, as well as on pressure condition, since, applying the fundamental laws of dynamics to the piston, its maximal velocity, $v_{\max }$ in $[\mathrm{m} / \mathrm{s}]$, can be expressed as:

$$
v_{\max }=\sqrt{\frac{2 P_{a t m} S h}{m_{p}}}
$$

Where $P_{a t m}$ represents the atmospheric pressure, $S$ the surface of the piston, $m_{p}$ its mass, and $h$ the impact height, all in SI units. The analysis of the velocity curves acquired clearly evidence a direct influence of impact height on the maximal velocity reached by the piston, which can be therefore directly extracted from these curves (Figure 3 a). Since this parameter, $V_{\max }$, can be considered as representative of the energy transmitted during the impact, it can be further correlated to the maximal pressure reached, $P_{\max }$ (Figure $3 \mathrm{~b}$ ).

The Joukowsky equation, derived from the water hammer theory [27], i.e. the hydrodynamic description of the flow of a fluid in a pipe being stopped at a time t, can be used to establish the correlation between $V_{\max }$ and $P_{\max }$ :

$$
\Delta P=\rho c \Delta v
$$

Where $\Delta P$ stands for the pressure difference $\left(P_{\max }-P_{a t m}\right), \rho$ represents the density of the liquid media, $c$ the speed of sound, and $\Delta v$ the difference between $V \max$ and the piston speed at $\mathrm{t}=0$, i.e. $v o=0 \mathrm{~m} / \mathrm{s}$. Taking into account that initially the piston is held by the electromagnet, thus its velocity at that instant equals zero, and that the pressure inside the reactor is fixed at around 20 mbar just before the release of the piston, according to eq. 2 a linear trend should define the variation of $P_{\max }$ as a function of $V_{\max }$. Therefore, the slope of 
this line should be equal to the product of liquid density and the speed of sound in the liquid, i.e. water1000 kg/m $\mathrm{m}^{3}$ and $1500 \mathrm{~m} / \mathrm{s}$, respectively.

Figure $3 \mathrm{~b}$ plots the values of $P_{\max }$ vs. $V \max$ measured during different experiments performed at different piston heights. These experimental values are not very far away from the theoretical trend predicted by the Joukowsky equation. Moreover, the fitting of the experimental data to a linear function allows thus the calculation of the slope of this line, yielding an estimation of the value of the speed of sound in water. In our case, the value found was $1526 \mathrm{~m} / \mathrm{s}$, i.e. $1.73 \%$ error with respect to the expected value of $1500 \mathrm{~m} / \mathrm{s}$. This fact corroborates the applicability of the water hammer model as a simple way to correlate the impact effect, in terms of $P_{\max }$, to the amount of energy communicated in this impact, related to $V \max$.

There are, however, other important parameters that vary depending not only on the impact height, but also on the configuration of the set-up, i.e. the use of a mobile bottom and different bottom pressures. Figure 4 a shows $P_{\max }$ as well as $P_{\min }$, the minimal pressure measured during the rarefaction period taking place right after the impact, as a function of impact height and for three experimental configurations: fixed bottom, moving bottom at a pressure of 2 bar, and moving bottom at a pressure of 4 bar.

The fact of liberating the bottom of the reactor and, moreover, adding an external pressure, makes the whole liquid column behave as a kind of a spring that, upon the impact will move from the bottom to the top and back, forcing as well the rebound of the upper main piston. In fact, $P_{\max }$ increases as expected as a function of the impact height, however, the differences are softened when using the moving bottom configuration, especially when working at 4 bars. At $20 \mathrm{~mm}$ impact height, the three configurations converge into the same value of $P_{\max }$. For such a strong impact, no further energy can be recuperated from the moving bottom and the spring-like behavior of the liquid column. Minimal - rarefaction - 
pressures, $P_{\min }$, also vary with impact height. Moreover, $P_{\min }$ are always smaller when using the moving bottom at 4 bar, reaching values close to -35 bar at $20 \mathrm{~mm}$ impact height. The spring-like movement of the liquid column results in bigger depressions adding more tension to the liquid that would be thus more prompt to spall. The values of deltaP, i.e. maximal pressure difference $P_{\max }-P_{\min }$, measured during the experiments and shown in Figure $4 \mathrm{~b}$ are just a consequence of these facts and are all the time higher when using the moving bottom at 4 bar. Note here that $P_{\min }$ is most of the time negative (rarefaction period), thus resulting in deltaP values well above $P_{\max }$. The maximal velocity attained by the piston in its fall is also depicted in Figure 4 b, as a function of impact height and experiment configuration. Higher values of $V_{\max }$ are always measured for the most intensive configuration, i.e. moving bottom at 4 bar. Using the fixed bottom configuration results in a deceleration of the piston by the air/vapor boundary layer on the surface of water, that cannot be further compressed/displaced by pushing the base of the cylindrical reactor.

The use of a moving bottom has moreover important consequences in terms of the rebound of the upper piston, since the shockwave departing from the bottom of the reactor is further pushed up, lifting the upper piston and causing a different rebound phenomena, in terms of time and intensity. When using the moving bottom configuration at 4 bar pressure, the upper piston rebound appears some milliseconds earlier as the impact height is decreased, as depicted in Figure 5. At the highest impact height the amplitude of the shock wave generated is too big and it propagates more slowly than a shock wave of smaller amplitude generated by a softer impact.

All the parameters mentioned before determine the type of cavitation phenomena, the distribution of bubble diameters and its evolution as a function of time, i.e. upon impact, rarefaction, rebound, etc. The use of the CDD camera allowed the direct visualization of the cavitation phenomena occurring in the bulk of the liquid. Several videos were acquired using 
a frame rate of $25000 \mathrm{fps}$ (frames per second). Figure 6 presents different snapshots of one of the videos recorded during a typical piston impact. Just before the release of the piston $(\mathrm{t}=-$ $1.928 \mathrm{~ms}$ ) the liquid contains already several small bubbles that are formed upon its degasification while pulling vacuum between the piston and the water surface. The presence of these bubbles is crucial, since they may act as cavitation nuclei. The piston is then released and finally impacts the liquid surface. The bubbles first disappear upon the impact, i.e. water is fully degased as due to pressure increase, and then grow, as a consequence of the rarefaction period that follows the impact (negative pressures period).

A cluster of bubbles appears as a consequence of this negative pressure period, that instigates cavitation. These bubbles change rapidly their diameter in the scale of only a few microseconds, until they finally violently collapse. Several softer cavitation events are continuously observed, moreover after the rebound of the piston, see for example the snapshots at $\mathrm{t}=8.272$ and $8.522 \mathrm{~ms}$. Figure 7 shows the evolution of bubble size during these cavitation phenomena. Formed initially in the bulk of a bubble cloud, the cavitation bubble grows and then shrinks periodically as the shock-waves created upon the impact and/or rebound of the piston propagate. The analysis of bubble size distribution during an impact remains difficult, since resonant bubbles of different sizes are simultaneously excited, due to the multi-frequency nature of the trail of shock-waves.

Figure 8 presents the results of a Daubechies-type wavelet analysis performed on the pressure signal acquired during an experiment performed at $20 \mathrm{~mm}$ height, using the moving bottom configuration at 4 bar. The Daubechies wavelet method (coded in C language) allows the determination of the frequency distribution corresponding to the pressure variations induced in the liquid media as a consequence of the propagation of the shock-waves generated by the piston impact. This kind of analysis is preferred to traditional Fast Fourier Transform (FFT) analysis when frequencies are multiple and distributed along a time scale, since it allow 
the complete characterization and reconstruction of a given signal in time and frequency [28]. The color scale in Figure 8 represents the normalized amplitudes, whereas the frequencies are plotted versus time. Two peaks can be clearly distinguished in this plot, appearing at the same time intervals than the main pressure peaks, respectively corresponding to piston impact and rebound. High frequencies thus appear when pressure increases in the liquid media, reaching maximal values around $100 \mathrm{kHz}$. Note there that the resonant frequency of the pressure sensors is $125 \mathrm{kHz}$, thus, the frequencies higher than this limit identified in the wavelet analysis are not quite reliable due to this fact, moreover taking into account that the raw pressure signal (non-filtered) was employed. High frequencies nevertheless correspond all the time to low amplitudes whereas the highest amplitudes - highest population - correspond to low frequencies, i.e. $<1 \mathrm{kHz}$, and occur in the time interval between impact and rebound, further corresponding to the continuous cavitation phenomena observed in the videos captured during the experiment, and depicted in Figure 7. The same wavelet analysis was performed for different impact heights and set-up configurations. The frequency distribution visibly changes when using either the fixed or the mobile bottom configuration at different pressures. However, differences are quite minimal, this allowing us to conclude that in the bulk of the liquid media, the trail of sock-waves created and its propagation are able to excite cavitation bubbles/nuclei in a wide range of frequencies between 0.1 and $100 \mathrm{kHz}$, thus confirming the multi-frequency character of this kind of impact-induced cavitation.

\subsection{Phenol oxidation in water media under shock-induced cavitation}

The first consequence of this multi-frequency type of cavitation will be thus the possibility of exciting the whole bulk of the liquid comprising bubbles of very different sizes. The collapse of bubbles will thus result in the formation of ultra high local temperatures and pressures, similar to the ones in the presence of classical ultrasounds. These phenomena are 
thought to contribute to the formation of free radicals in a water media, which can be finally consumed in oxidation of organic pollutants in wastewaters.

In order to explore the intensification of the oxidation of organic pollutants under shock-induced sonochemical conditions, the impact experiments were performed introducing an aqueous solution containing either 10 or $50 \mathrm{mg} / \mathrm{L}$ of phenol in the cylindrical reactor. The piston was thus held at either 8,15 or $20 \mathrm{~mm}$ over the surface of the liquid and released several times, up to 1000 successive impacts. The content of phenol in the treated solution was periodically analyzed by gas chromatography and total organic carbon (TOC). Let us note here that the free radical reaction was quenched through the addition of $\mathrm{MnO}_{2}$ and the resulting samples were filtered prior to their analysis.

Figure 9 contains the results obtained in the first set of experiments, performed using two different phenol concentrations, 10 and $50 \mathrm{mg} / \mathrm{L}$, and at two different impact heights, 20 and $15 \mathrm{~mm}$. Even in the sole presence of deionized water in the reaction media, shockinduced cavitation is able to promote phenol oxidation. Though the final extent of phenol degradation only amounts to $7 \%$, this points directly to the creation of free radicals in the water media as a consequence of the piston impact, able to attack phenol molecules. The concentration of such free radicals might be however relatively low in comparison to the initial concentration of the phenol solution, as evidenced by the slower degradation of the solution containing $50 \mathrm{mg} / \mathrm{L}$ instead of $10 \mathrm{mg} / \mathrm{L}$. However, TOC analysis showed almost zero mineralization, pointing to phenol degradation resulting in the formation of intermediate oxidation products, such as carboxylic acids. This was further evidenced by the appearance of several peaks in the analysis chromatogram at 1.5-1.8 $\mathrm{s}$ retention time, with phenol peak appearing between 3.25-3.5 s. 
A chemical oxidant, $\mathrm{H}_{2} \mathrm{O}_{2}$ (30 wt.\% solution) was then added to the reaction media, in order to boost the generation of free radicals. Figure 10 shows the results obtained in terms of phenol degradation, when adding either 5 or $65 \mathrm{~mL}$ of the $\mathrm{H}_{2} \mathrm{O}_{2}$ solution. Figures 10 a and 10 b show as well the results of phenol degradation in the absence of the shock-induced cavitation phenomena, i.e. using the same reactor volume, the same amount of $\mathrm{H}_{2} \mathrm{O}_{2}$, under conventional stirring at ambient temperature. Phenol degradation reaches $15 \%$ after 1000 impacts, i.e. 120 minutes of reaction, when adding only $5 \mathrm{~mL}$ of the $\mathrm{H}_{2} \mathrm{O}_{2}$ solution. When adding $65 \mathrm{~mL}$ of $\mathrm{H}_{2} \mathrm{O}_{2} 30$ wt.\%, degradation upon 1000 impacts reaches $61 \%$, whereas $14 \%$ degradation was measured in the absence of shock-induced cavitation, i.e. under conventional stirring. These results further support the fact of increased free radical formation as a consequence of piston impact, either due to the enhanced hydrolysis of $\mathrm{H}_{2} \mathrm{O}_{2}$ to generate these active species [29], or to the favoring of the phenol oxidation reaction in their presence, or maybe to the simultaneous intensification of both processes. The percentages of mineralization determined from the TOC analysis were all the time around $25-35 \%$ lower than the phenol degradation values determined by means of gas chromatography. The peaks at low retention times were all the time observed, confirming the formation of oxidation intermediates.

Moreover, the phenol solutions treated with 1000 impacts were left inside the reactor and analyzed 80 minutes after the treatment. Final phenol degradation extents - upon 200 min reaction time - of 84.2 and $92.3 \%$ were measured for the reactions impacted with an initial position of the piston of 20 and $8 \mathrm{~mm}$, respectively, corresponding to TOC elimination of 45.3 and $56.2 \%$. Such high degradation extents clearly evidence a substantial acceleration of the oxidation of phenol in aqueous solution, assisted by the presence of the shock-induced cavitation phenomena. The comparison of the results obtained in presence and in absence of $\mathrm{H}_{2} \mathrm{O}_{2}$ point to phenol oxidation preferably occurring through a radical-formation route 
involving the generation of hydroxyl radicals, rather than through simple air induced oxidation, i.e. pyrolysis route. In this sense, we believe that the mechanisms behind phenol oxidation under shock-induced cavitation conditions may be very similar to typical ultrasound sonochemistry, with two different types of acoustical phenomena taking place, i.e. the propagation of a shock wave and an ultrasonic wave in each case.

In fact, the differences observed between the reaction extents measured upon the impact at different piston initial positions are directly related to the particular features of the cavitation induced in each case, such as the maximal cavitation bubble diameter, limited by the rebound of the piston, and the co-existence of different cavitation regimes of different intensity. Further studies are needed in order to quantify the amount of active radicals created under different cavitation conditions and thus to determine their influence in the acceleration of the oxidation of organic pollutants in aqueous solutions. Therefore it seems that the amount of total energy provided to the system, controlled by the impact height, or the maximal pressure attained are not the key influencers. The control of bubble size growth and collapse through the propagation of the shock-waves is preponderant, this can be observed through the faster oxidation rate measured at $8 \mathrm{~mm}$ rather than at $20 \mathrm{~mm}$. Moreover, the duration of the treatment needs to be optimized, i.e. in terms of number of impacts. This implies a thoughtful study of the degradation mechanisms involved, which is been currently overtaken by means of the quantification of the amount of radicals formed in the reaction media. Reducing the number of impacts while achieving the same mineralization extent may result in important energy savings.

\section{Conclusions}

The cavitation phenomena induced by means of the impact of a solid piston on a liquid surface was considered and applied for the acceleration of phenol oxidation in aqueous media. 
The impact intensity, as well as the energy communicated in the impact, were directly related to the impact height. This proportionality was verified using a simple water hammer model. Reactor configuration, i.e. fixed or moving bottom, moreover conditioned the type of phenomena observed upon the piston impact. In this way, setting the bottom of the reactor free resulted in a spring-like behavior of the liquid column that enhanced the propagation of the induced shock-waves.

The rarefaction period observed upon the piston impact was the origin of several violent cavitation phenomena occurring in a cloud of bubbles having very different diameters. The collapse of these bubbles finally resulted in a complete rupture of the liquid, as visualized with the CDD camera. After the main impact and rarefaction period, several cavitation effects, less violent, were continuously observed, specially as a consequence of piston rebound, which was further favored when using the moving bottom configuration. The wavelet analysis of the pressure signals recorded in the liquid media confirmed the multi-frequency nature of this shock-induced cavitation and evidenced the possibility of exciting a wide range of frequencies between $1 \mathrm{kHz}$ and $100 \mathrm{kHz}$ in a very short time interval.

Phenol degradation was found to be possible in the presence of impact-induced cavitation, even in the absence of any oxidizing agent. However, no mineralization was measured in this case. In order to achieve acceptable mineralization extents, $\mathrm{H}_{2} \mathrm{O}_{2}$ was added to the reaction mixture. A considerable acceleration of the oxidation reaction was then observed, with TOC reductions reaching 45.3-56.2\% upon less than 2 hours total reaction time. This fact was linked to the enhancement of $\mathrm{H}_{2} \mathrm{O}_{2}$ hydrolysis leading to the formation of a high concentration of active radical species in the liquid media, and to the acceleration of their subsequent reaction with phenol molecules, under shock-induced cavitation conditions.

\section{References}


[1] C. Comninellis, A. Kapalka, S. Malato, S. A. Parsons, I. Poulios, I., D. Mantzavinos, Advanced oxidation processes for water treatment: advances and trends for R\&D, J. Chem. Technol. Biotechnol. 83 (2008) 769-776.

[2] R. Andreozzi, V. Caprio, A. Insola, R. Marotta, Advanced oxidation processes (AOP) for water purification and recovery, Catal. Today 53 (1999) 51-59.

[3] Y. G. Adewuyi, Sonochemistry in environmental remediation. 1. Combinative and hybrid sonophotochemical oxidation processes for the treatment of pollutants in water. Environ. Sci. Technol. 39 (2005) 3409-3420.

[4] Gogate, P. R., Treatment of wastewater streams containing phenolic compounds using hybrid techniques based on cavitation: a review of the current status and the way forward. Ultrason. Sonochem. 15 (2008) 1-15.

[5] N.M. Navarro, T. Chave, P. Pochon, I. Bisel, S.I. Nikitenko, Effect of ultrasonic frequency on the mechanism of formic acid sonolysis, J. Phy. Chem. B 115 (2011) 2024-2029.

[6] T. Chave, N.M. Navarro, P. Pochon, N. Perkas, A. Gedanken, S.I. Nikitenko, Sonocatalytic degradation of oxalic acid in the presence of oxygen and Pt/TiO2, Cat. Today (2015) 55-62.

[7] P.R. Gogate, Cavitational reactors for process intensification of chemical processing applications: a critical review, Chem. Eng. Process Process Intensification 47 (2008): 515-527. [8] M.V. Bagal, P.R. Gogate, Wastewater treatment using hybrid treatment schemes based on cavitation and Fenton chemistry: A review, Ultrason. Sonochem. 21 (2014) 1-14.

[9] N. Golash, P.R. Gogate, Degradation of dichlorvos containing wastewaters using sonochemical reactors, Ultrason. Sonochem. 19 (2012) 1051-1060. 
[10] P.R. Gogate, S. Mujumdar, A. B. Pandit, A sonochemical reactor for the removal of formic acid from wastewater, Ind. Eng. Chem. Res. 41 (2002) 3370-3378.

[11] T.G. Leighton, The Acoustic Bubble, Academic Press, London, 1994.

[12] C.E. Brennen, Cavitation and bubble dynamics. Cambridge University Press, 2013.

[13] Y.T. Shah, A.B. Pandit, V.S. Moholkar, Cavitation reaction engineering. Springer Science \& Business Media, 2012.

[14] S.I. Nikitenko, F. Chemat, Ultrasound in process engineering, Green Process Engineering: From concepts to industrial applications, 2015, pp. 145.

[15] K.S. Suslick, D.A. Hammerton, R.E. Cline, Sonochemical hot spot, J. Amer. Chem. Soc. 108 (1986) 5641-5642.

[16] G. Cum, G. Galli, R. Gallo, A. Spadaro, Role of frequency in the ultrasonic activation of chemical reactions, Ultrasonics, 30 (1992) 267-270.

[17] M.H. Entezari, P. Kruus, Effect of frequency on sonochemical reactions. I: Oxidation of iodide, Ultrason. Sonochem., 1 (1994) S75-S79.

[18] T.J. Mason, A.J. Cobey, J.E. Graves, D.Morgan, New evidence for the inverse dependence of mechanical and chemical effects on the frequency of ultrasound, Ultrason. Sonochem., 18 (2011) 226-230.

[19] J. Rooze, E.V. Rebrov, J.C. Schouten, J.T.F. Keurentjes, Effect of resonance frequency, power input, and saturation gas type on the oxidation efficiency of an ultrasound horn, Ultrason. Sonochem., 18 (2011) 209-215.

[20] L.H. Thompson, L.K. Doraiswamy, Sonochemistry: science and engineering, Ind. Eng. Chem. Res., 38 (1999) 1215-1249. 
[21] P.R. Gogate, V.S. Sutkar, A.B. Pandit, Sonochemical reactors: important design and scale up considerations with a special emphasis on heterogeneous systems, Chem. Eng. J., 166 (2011) 1066-1082.

[22] V.S. Sutkar, P.R. Gogate, Design aspects of sonochemical reactors: techniques for understanding cavitational activity distribution and effect of operating parameters, Chem. Eng. J., 155 (2009) 26-36.

[23] P.R. Gogate, A.B. Pandit, Sonophotocatalytic reactors for wastewater treatment: a critical reviewAIChE J., 50 (2004) 1051-1079.

[24] N.N. Mahamuni, Y.G. Adewuyi, Advanced oxidation processes (AOPs) involving ultrasound for waste water treatment: a review with emphasis on cost estimation, Ultrason. Sonochem., 17 (2010) 990-1003.

[25] Q.X. Wang, Underwater explosion bubble dynamics in a compressible liquid. Phys. Fluids, 25 (2013) 072104.

[26] T.W. Randolph, E. Schiltz, D. Sederstrom, D. Steinmann, O. Mozziconacci, C. Schöneich, E. Freund, M.S. Ricci, J.F. Carpenter, C.S. Lengsfeld, Do not drop: mechanical shock in vials causes cavitation, protein aggregation, and particle formation, J. Pharm. Sci. 104 (2015) 602-611.

[27] N. Joukowsky, Über den hydraulischen Stoss in Wasserleitungsrohren, St. Petesbourg, 1900.

[28] V. Brani, P. Mueller, Wavelets for kids, Duke’s Univesity, 1994. 
[29] R. Chand, N.H. Ince, P.R. Gogate, D.H. Bremner, Phenol degradation using 20, 300 and $520 \mathrm{kHz}$ ultrasonic reactors with hydrogen peroxide, ozone and zero valent metals, Sep. Purif. Technol. 67 (2009) 103-109. 
Captions to Figures

Figure 1. Shock-induced cavitation set-up, schematic representation of piston impact.

Figure 2. Time evolution of pressure signals (top and bottom), as well as piston velocity, upon release and impact.

Figure 3. a) Velocity as a function of time registered during a piston impact, fixed bottom; and b) Pmax versus Vmax, experimental data, water hammer model and linear fit.

Figure 4. a) Pmax and Pmin, and b) delta $\mathrm{P}$ and vmax, as a function of impact height and experiment configuration: fixed, moving 2 bar and moving 4 bar bottom.

Figure 5. Pressure vs. time profiles for the experiments performed at 20,16, 12 and $8 \mathrm{~mm}$, moving bottom 4 bar: Rebound appearing sooner for smaller impact height.

Figure 6. Different snapshots of the video recorded with the aid of the CDD camera during a typical impact, showed along the pressure-time curve acquired for this experiment.

Figure 7. Detail of the cavitation phenomena induced upon piston rebound, evolution of the bubble cloud and bubble diameter during cavitation.

Figure 8. Wavelet analysis of frequency distribution during a $20 \mathrm{~mm}$ impact, moving bottom 4 bar experiment.

Figure 9. Evolution of phenol concentration with the number of impacts and time, during the experiments performed in the sole presence of shock-induced cavitation (no oxidants added).

Figure 10. Phenol degradation experiments in the presence of a) $5 \mathrm{~mL} \mathrm{H} 2 \mathrm{O} 230 \mathrm{wt} . \%$ solution and b) $65 \mathrm{~mL} \mathrm{H} 2 \mathrm{O} 230$ wt.\% solution, dashed lines: no cavitation, continuous line: shockinduced cavitation up to 1000 impacts. 


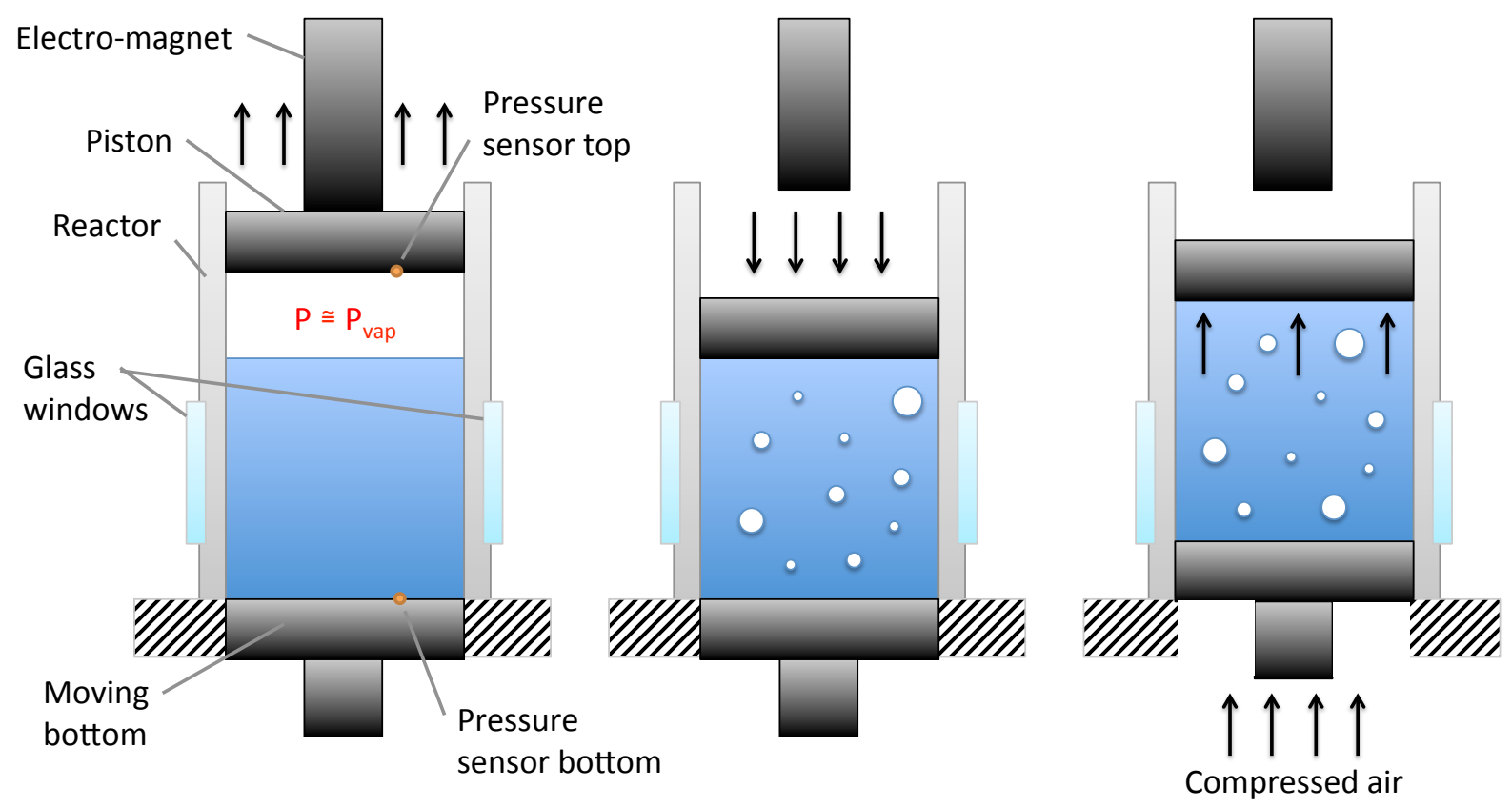

Figure 1. Shock-induced cavitation set-up, schematic representation of piston impact. 


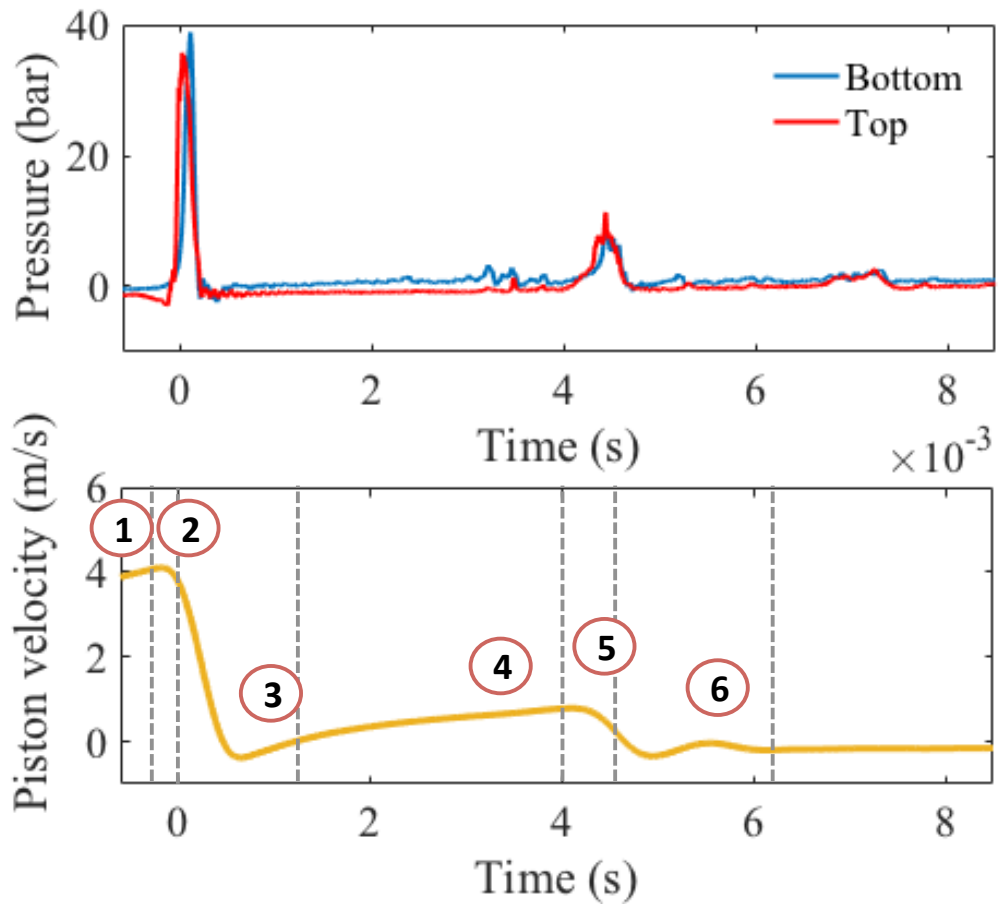

Figure 2. Time evolution of pressure signals (top and bottom), as well as piston velocity, upon release and impact. 

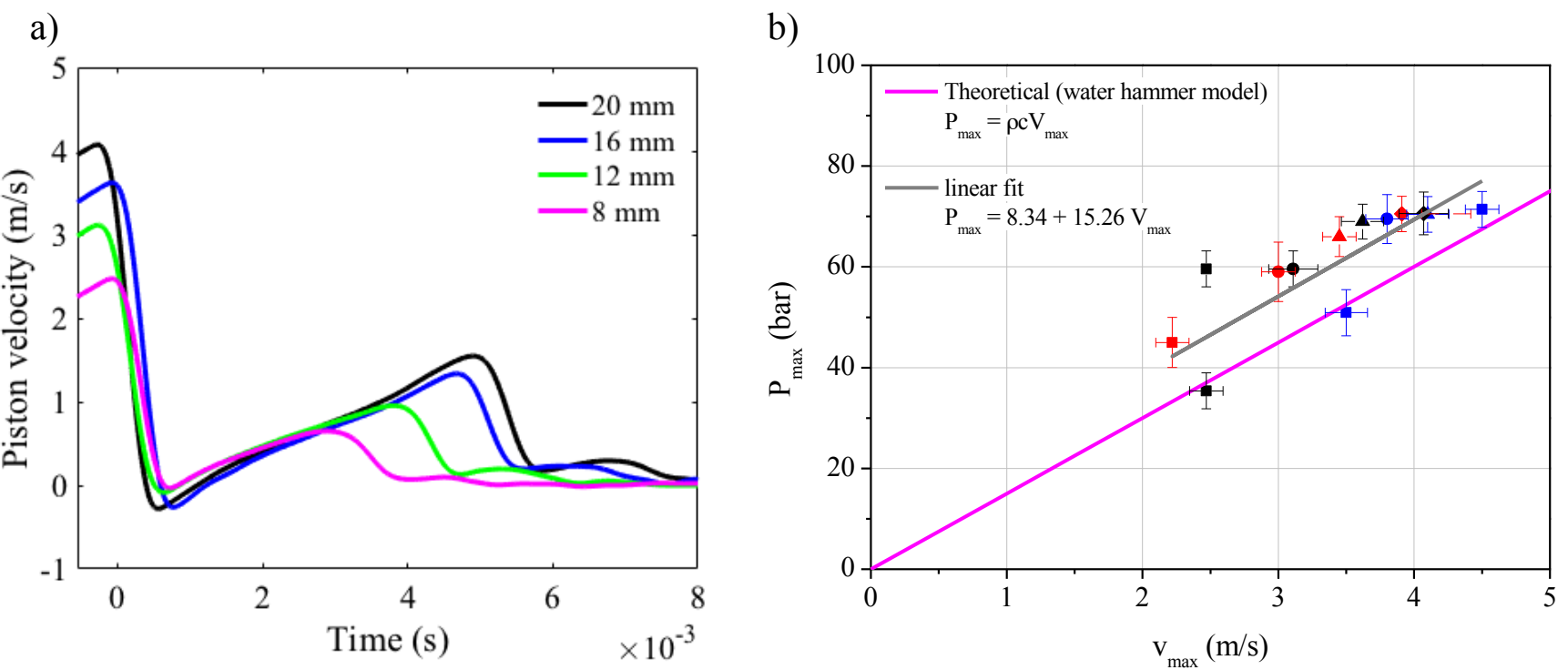

Figure 3. a) Velocity as a function of time registered during a piston impact, fixed bottom; and b) Pmax versus Vmax, experimental data, water hammer model and linear fit. 
a)

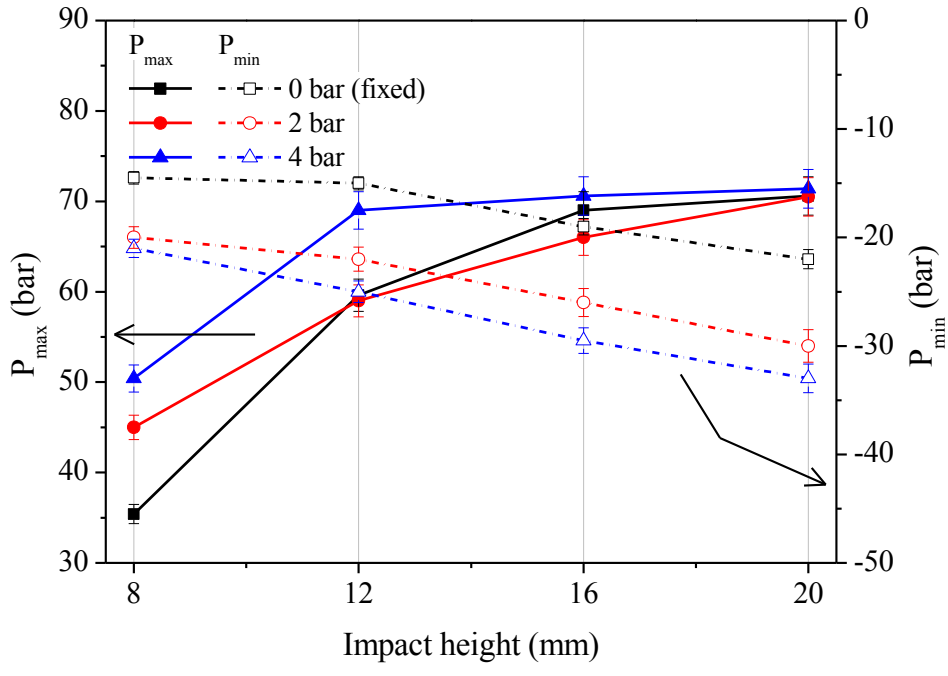

b)

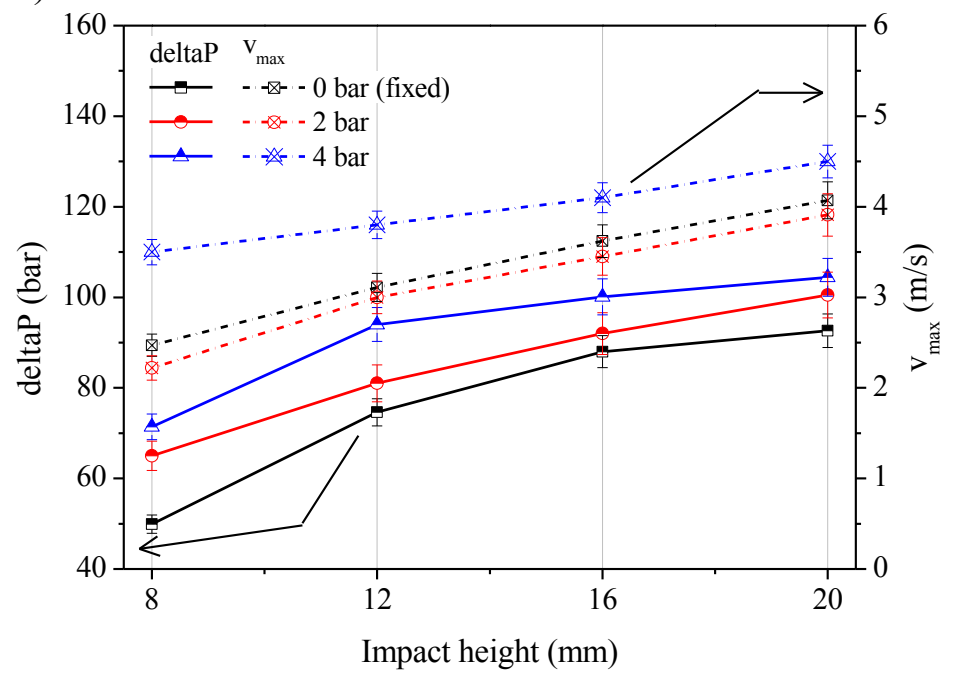

Figure 4. a) $P_{\max }$ and $P_{\min }$, and b) delta $P$ and $V_{\max }$ as a function of impact height and experiment configuration: fixed, moving 2 bar and moving 4 bar bottom. 


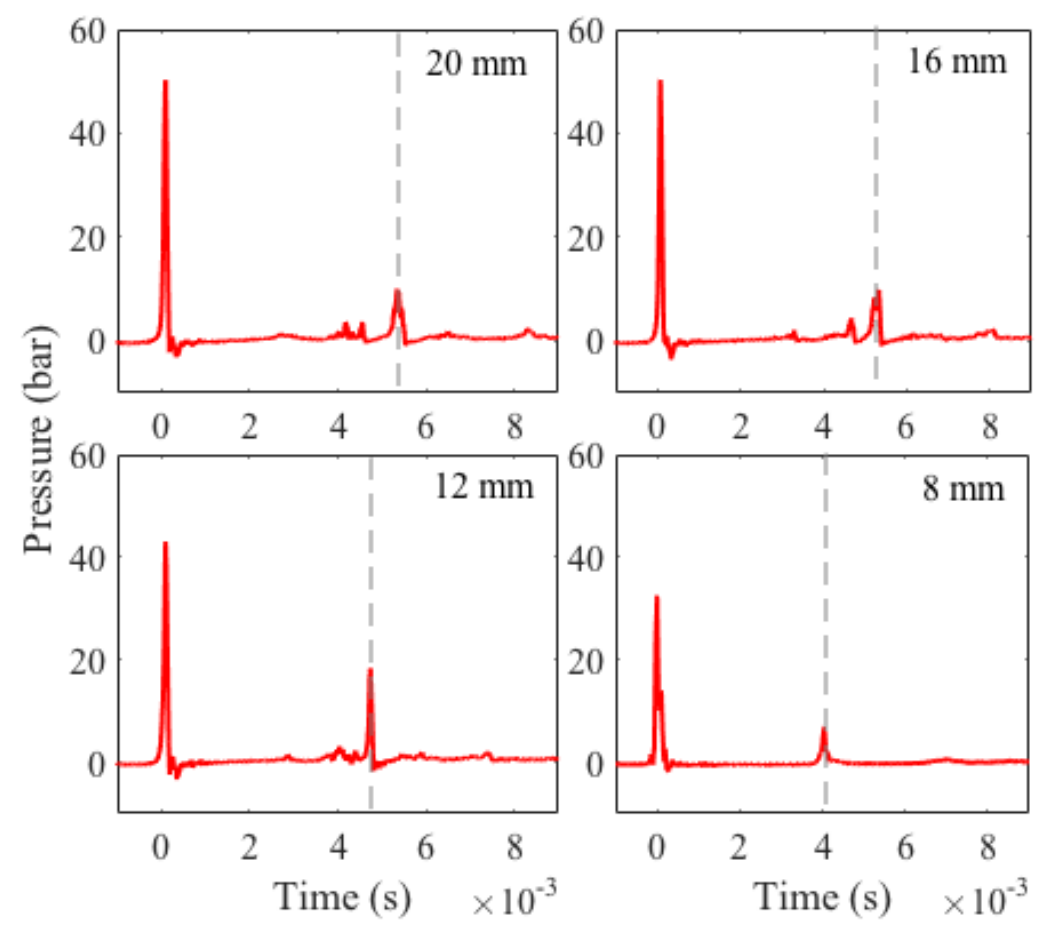

Figure 5. Pressure vs. time profiles for the experiments performed at 20,16, 12 and $8 \mathrm{~mm}$, moving bottom 4 bar: Rebound appearing sooner for smaller impact height. 


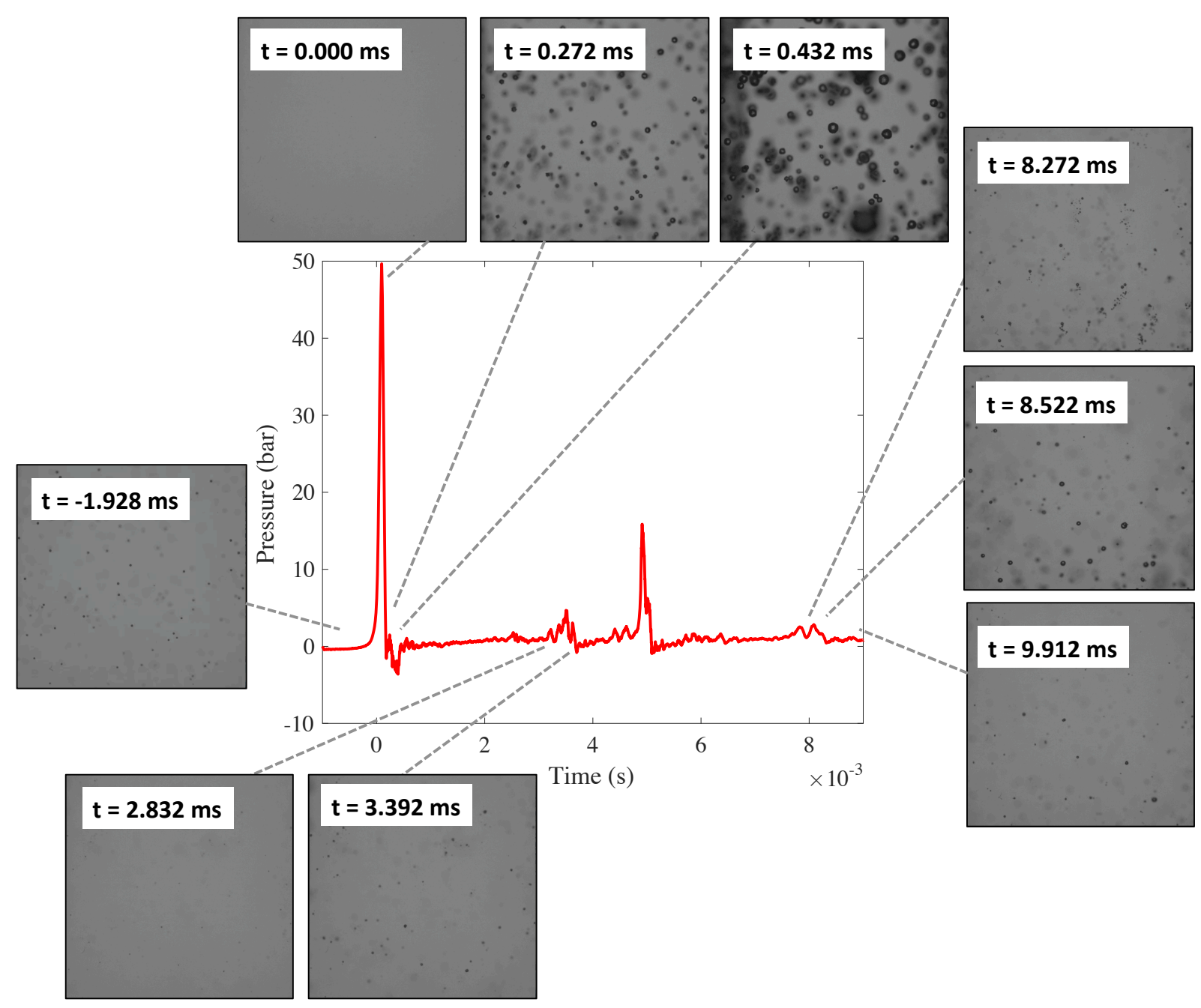

Figure 6. Different snapshots of the video recorded with the aid of the CDD camera during a typical impact, showed along the pressure-time curve acquired for this experiment. 


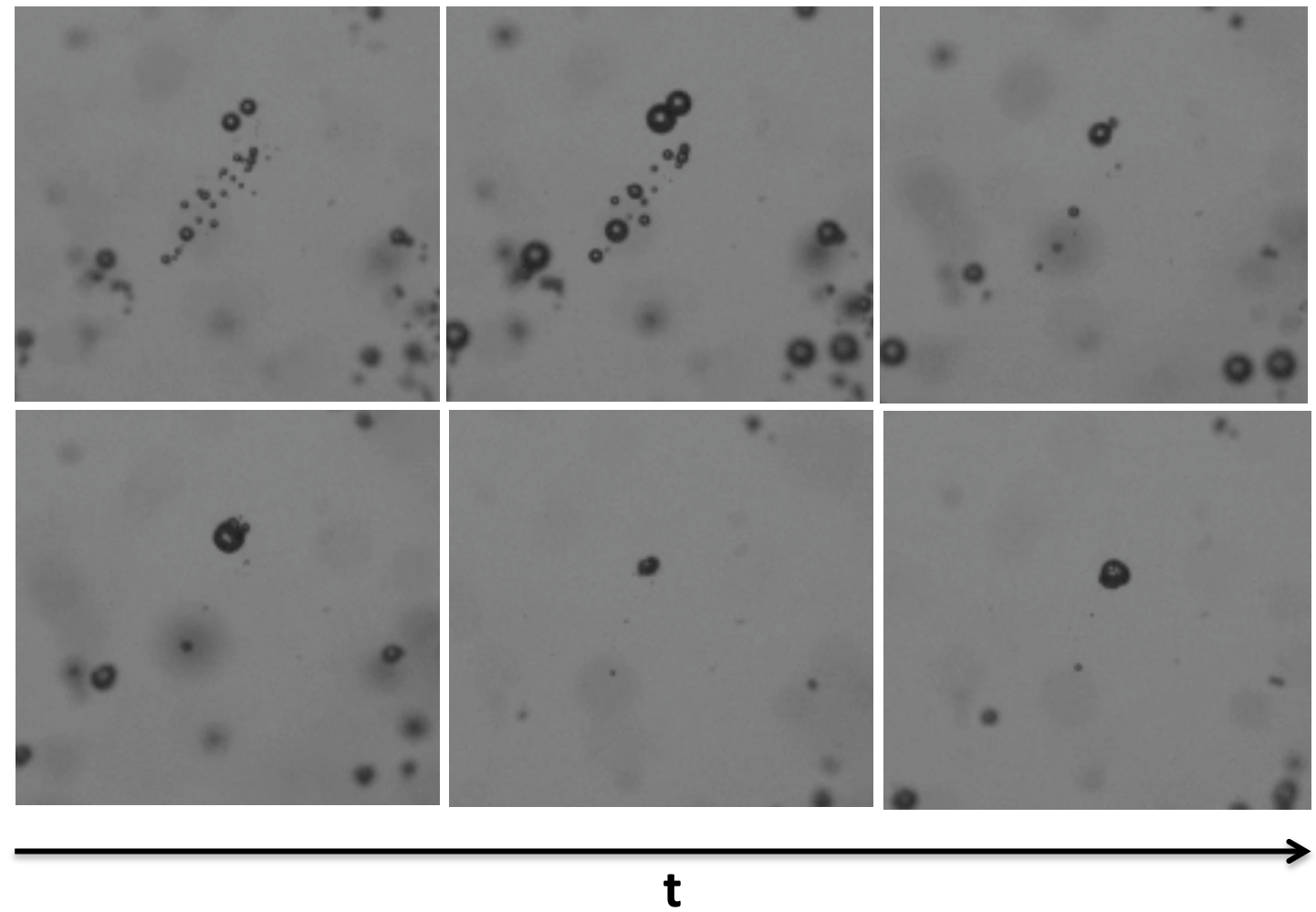

Figure 7. Detail of the cavitation phenomena induced upon piston rebound, evolution of the bubble cloud and bubble diameter during cavitation. 


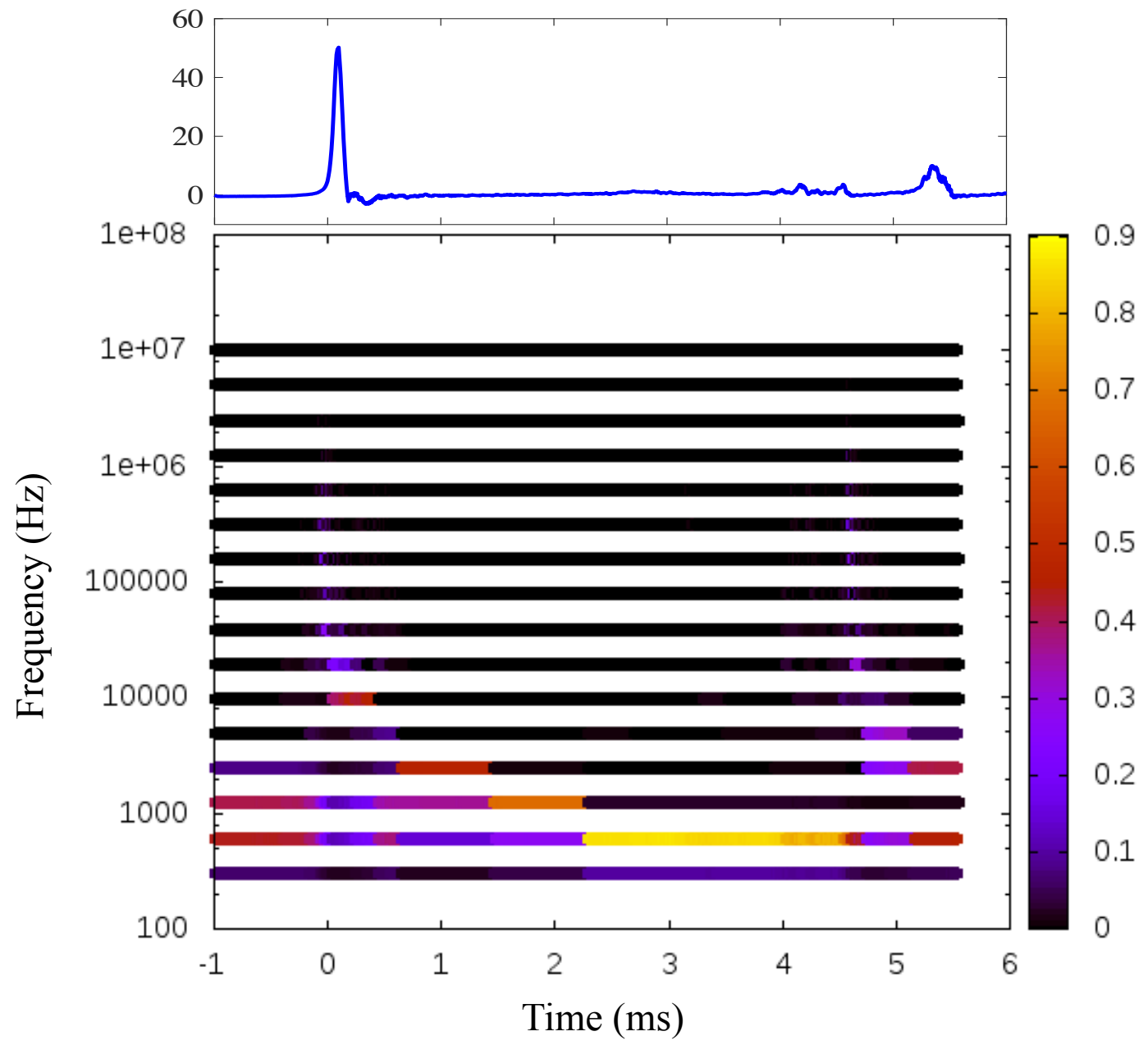

Figure 8. Wavelet analysis of frequency distribution during a $20 \mathrm{~mm}$ impact, moving bottom 4 bar experiment. 


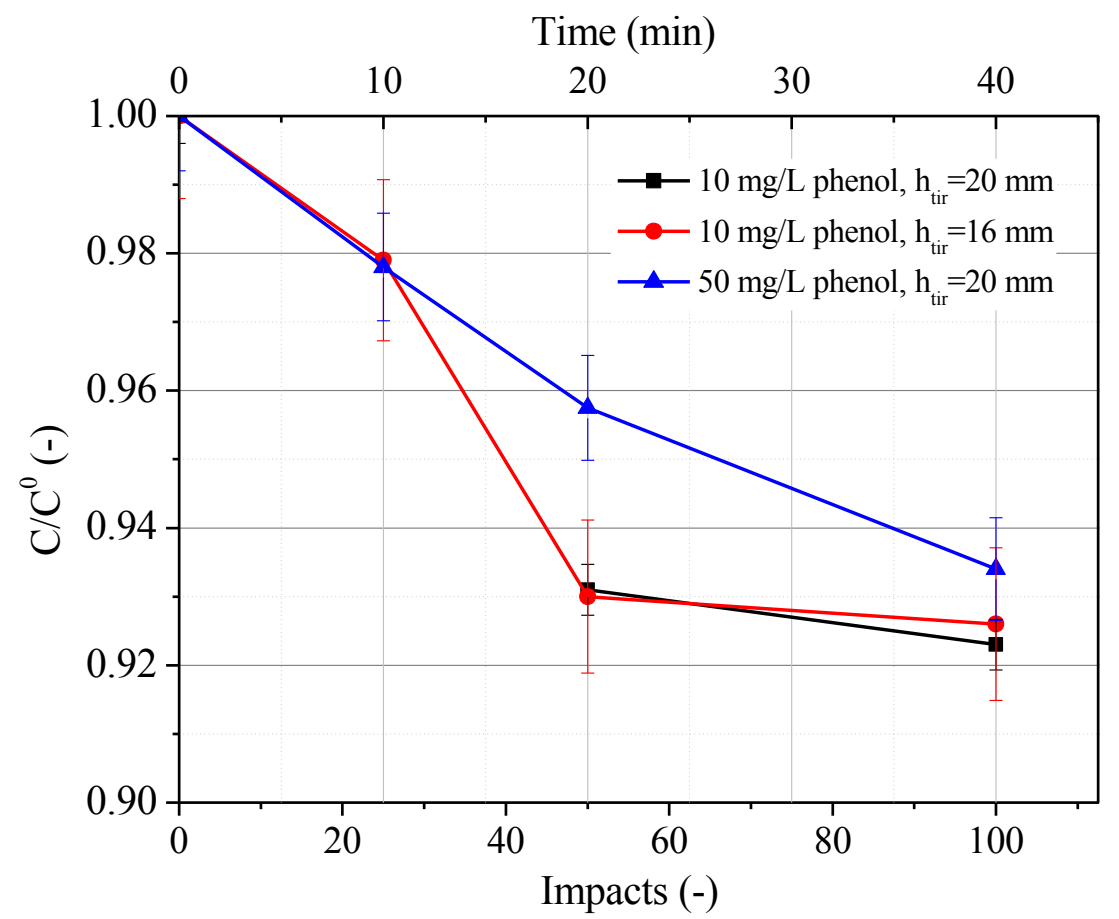

Figure 9. Evolution of phenol concentration with the number of impacts and time, during the experiments performed in the sole presence of shock-induced cavitation (no oxidants added). 
a)

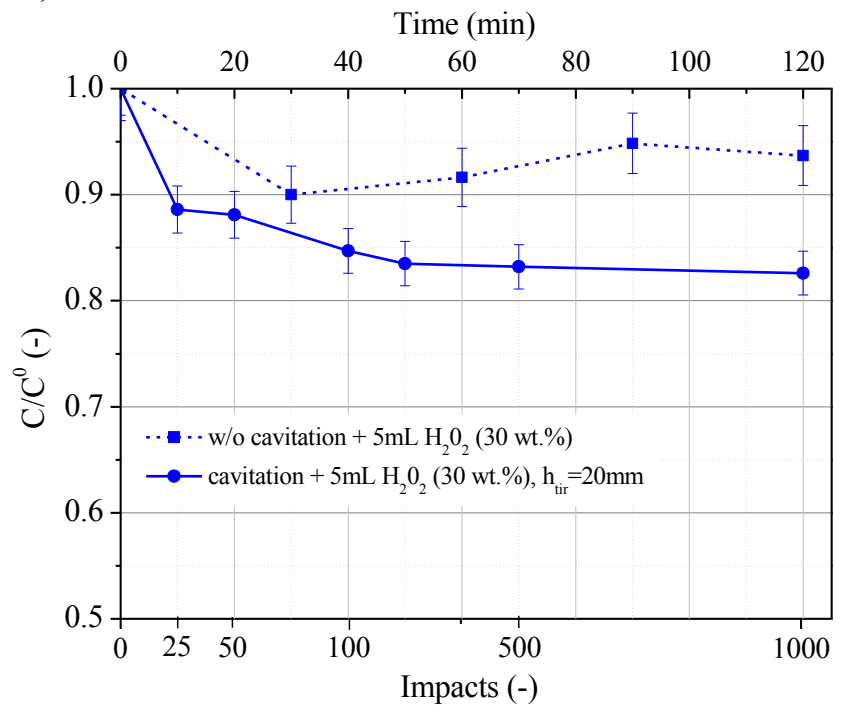

b)

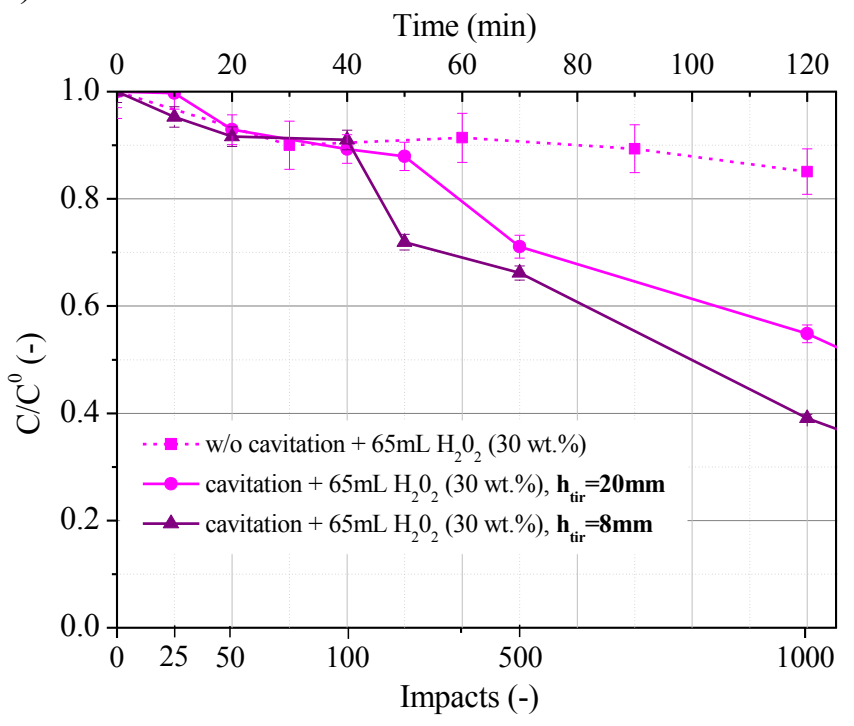

Figure 10. Phenol degradation experiments in the presence of a) $5 \mathrm{~mL} \mathrm{H}_{2} \mathrm{O}_{2} 30 \mathrm{wt} \%$ solution and b) $65 \mathrm{~mL} \mathrm{H}_{2} \mathrm{O}_{2} 30 \mathrm{wt} . \%$ solution, dashed lines: no cavitation, continuous line: shockinduced cavitation up to 1000 impacts. 